\title{
Probelab ReImager: Open Source Software for Streamlining Image Processing
}

Noah $\mathrm{Kraft}^{1}$ and Anette von der Handt ${ }^{2}$

${ }^{1}$ University of Minnesota, Bemidji, Minnesota, United States, ${ }^{2}$ University of Minnesota, Minneapolis, Minnesota, United States

Most commercial vendor-supplied software excels in acquisition and processing of data but falls short on customization and batch processing options and/or support for modern imaging formats. Export and customization of these images in third-party software is time consuming and adds to the workload to achieve publishable products for scientific journals. In our case, we use Thermo Scientific's Pathfinder and its predecessor NSS software to capture images on our JEOL JXA8530FPlus electron microprobe. While this software offers data export to various report templates, it lacks a batch export option for images in their native resolution. Furthermore, it does not allow for visual customization.

To fill this gap, we have created the program "Probelab ReImager", which facilitates easy batch exportation and customization - including annotations - of electron images and X-ray intensity maps. To create an easy to use and well supported program, we wrote Probelab ReImager in NodeJS utilizing Electron, resulting in a mobile and flexible web browser-based desktop application. Probelab Reimager is currently supported and released on Windows 7, 8, and 10, and release is planned for both Unix-based operating systems and MacOS. Distribution is through a central web domain with an API that identifies new updates or static major, minor, and bugfix releases. While NodeJS is not extremely efficient with handling large datasets such as images, it offers a large community supported set of open source repositories and is easier to maintain and expand than similar languages like Python. To provide fast processing of images without lag or "freezing" during use, an average of 300MB of memory is required across several processes. On an average Intel i5 $5^{\text {th }}$ gen CPU it fully processes, customizes, and exports a 4096-resolution image in less than 20 seconds, with mobile processors taking up to 30 seconds.

Probelab ReImager can add and customize a multitude of image annotations, quickly process and then batch export images (Figure 1). Current features include: customizable scale bar location, size, length, and background options; analysis positions can be displayed or hidden, colored for optimal contrast; form and size of position markers can also be customized (Figure 2). For X-ray intensity maps, the Probelab ReImager allows customization of color scales, opacity and order of display on either the electron image or a solid background. Image settings and their customization can be uniquely stored and processed when exported allowing for different settings for each exported image.

While many programs still only export images as TIFFs, this archaic image format has been superceded by formats such as PNG for lossless and JPG for lossy compression. Both of these formats offer more advanced metadata and compression, and are supported on every operating system. To facilitate the change of image formats, Probelab ReImager by default exports images to PNG but also supports JPG, WebP, and TIFF.

Probelab ReImager is released to the public and available at https://reimager.probelab.net. While currently limited to data acquired by Thermo Scientific's Pathfinder and NSS software, we are currently working on support for images acquired with JEOL EPMA and SEM-platform software, Cameca's "PeakSight" and Probe Software Inc.'s "Probe for EPMA". Support for Bruker, Oxford, and other software vendors is anticipated [1]. 


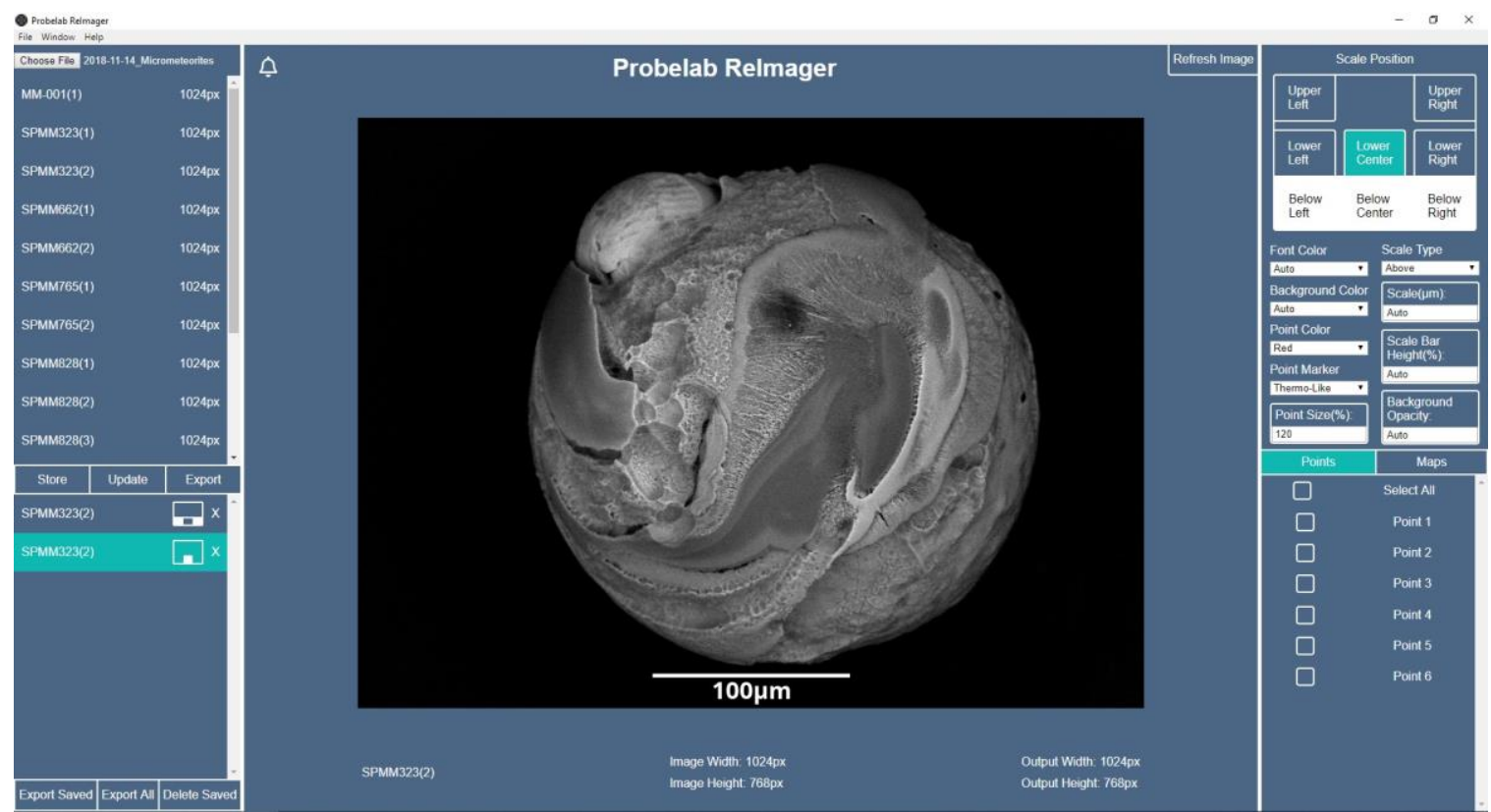

Figure 1. Graphical user interface of Probelab ReImager.

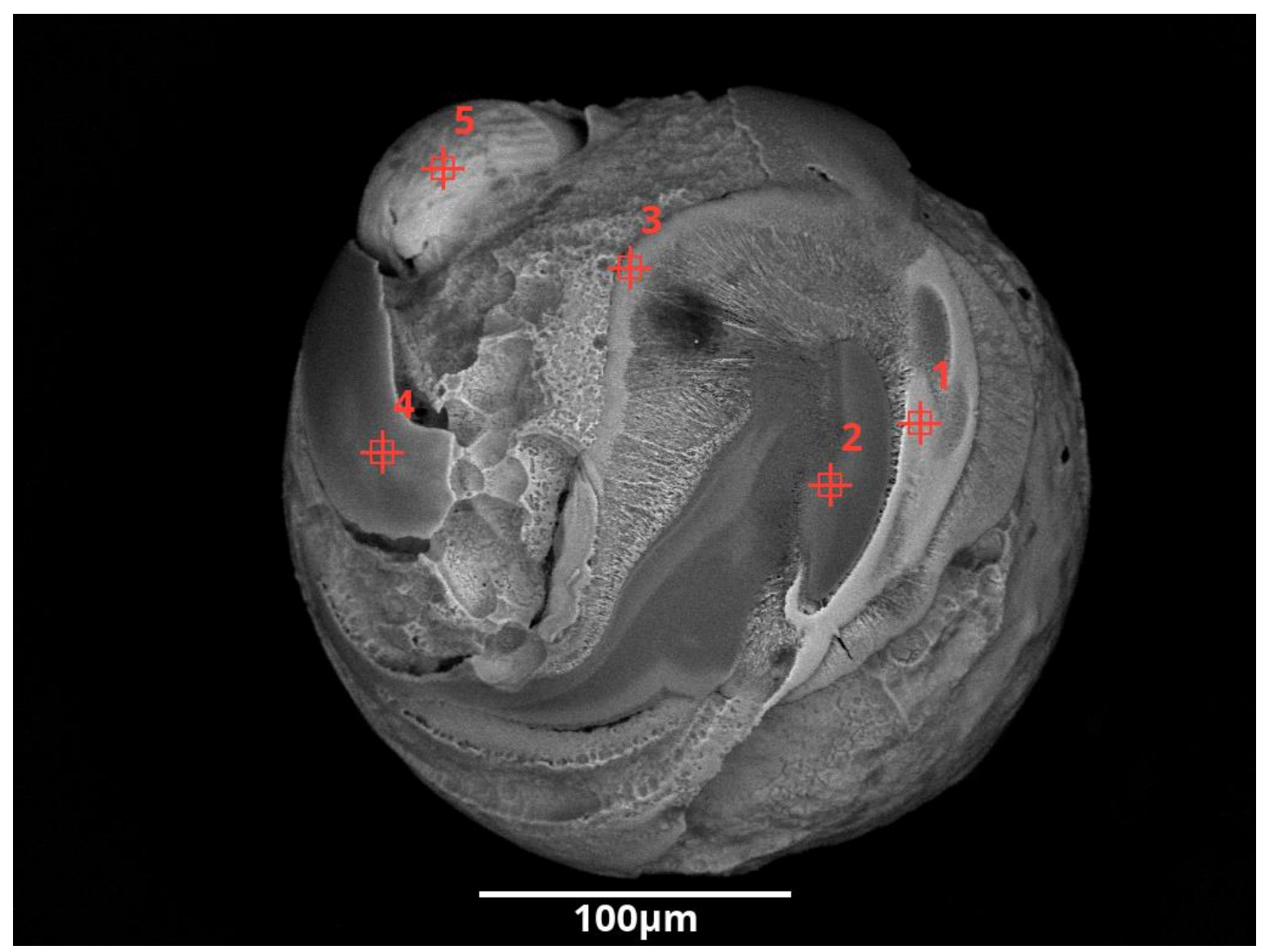

Figure 2. Example of image output with image scale bar and analysis positions. Image annotations can be fully customized for size, color and position.

\section{References}

[1] Support for this research came from the National Science Foundation: EAR-1849465 (AVDH). 